\title{
Synchrotron Studies of the First-Order Melting Transitions of Hexatic Monolayers and Multilayers in Freely Suspended Liquid-Crystal Films
}

\section{Citation}

Amador, S., Peter S. Pershan, H. Stragier, B. D. Swanson, D. J. Tweet, L. B. Sorensen, E. B. Sirota, G. E. Ice, and A. Habenschuss. 1989. Synchrotron studies of the first-order melting transitions of hexatic monolayers and multilayers in freely suspended liquid-crystal films. Physical Review A 39(5): 2703-2708.

\section{Published Version}

doi:10.1103/PhysRevA.39.2703

\section{Permanent link}

http://nrs.harvard.edu/urn-3:HUL.InstRepos:10357467

\section{Terms of Use}

This article was downloaded from Harvard University's DASH repository, and is made available under the terms and conditions applicable to Other Posted Material, as set forth at http:// nrs.harvard.edu/urn-3:HUL.InstRepos:dash.current.terms-of-use\#LAA

\section{Share Your Story}

The Harvard community has made this article openly available.

Please share how this access benefits you. Submit a story.

Accessibility 


\title{
Synchrotron studies of the first-order melting transitions of hexatic monolayers and multilayers in freely suspended liquid-crystal films
}

\author{
S. Amador and P. S. Pershan \\ Division of Applied Sciences and Department of Physics, Harvard University, \\ Cambridge, Massachusetts 02138
}

H. Stragier, B. D. Swanson, D. J. Tweet, and L. B. Sorensen

Department of Physics, FM-15, University of Washington, Seattle, Washington 98195

E. B. Sirota

Exxon Research and Engineering Co., Corporate Research Science Laboratories, Route 22 East, Annandale, New Jersey 08801

G. E. Ice

Metals and Ceramics Division, Oak Ridge National Laboratory, P.O. Box 2008, Oak Ridge, Tennessee 37831

A. Habenschuss

Chemistry Division, Oak Ridge National Laboratory, P.O. Box 2008, Oak Ridge, Tennessee 37831

(Received 26 September 1988)

\begin{abstract}
Synchrotron $x$-ray diffraction has been used to study the surface and the interior hexatic-to-liquid (smectic-I to smectic-C) melting transitions of freely suspended liquid-crystal films of $N$-[4'-( $n$-heptyl)benzylidene]-4-( $n$-heptyl)aniline (70.7) five molecular layers thick. Both the surface hexatic monolayers and the interior hexatic layers melt via hysteretic first-order transitions. After the two surface layers undergo a first-order transition to the smectic-I phase, the surface hexatic correlation length $\xi$ evolves smoothly from $\sim 100$ to $\sim 300 \AA$ with a roughly square-root form, $\xi \sim\left|T-T_{c}\right|^{1 / 2}$.
\end{abstract}

\section{INTRODUCTION}

There has been intense interest in the possibility of continuous dislocation-mediated two-dimensional (2D) melting transitions in which a $2 \mathrm{D}$ crystalline phase melts into a $2 \mathrm{D}$ liquid via an intermediate $2 \mathrm{D}$ hexatic phase. ${ }^{1-3}$ This melting is predicted to occur through two continuous Kosterlitz-Thouless (KT) transitions ${ }^{4}$ - one between the crystal and the hexatic and another between the hexatic and the liquid. The predicted hexatic phases and the associated KT transitions stimulated many experimental searches for this behavior in physisorbed monolayers, ${ }^{5}$ in colloidal suspensions, ${ }^{6}$ and in freely suspended liquidcrystal (FSLC) films. ${ }^{7-10}$ Although phases with hexatic symmetry have been found in all three systems, the origin of the hexatic order and its relationship to the Kosterlitz-Thouless-Halperin-Nelson-Young (KTHNY) dislocation-mediated melting predictions are still unresolved and controversial. ${ }^{11-13}$ For example, most of the physisorbed monolayer studies have used substrates with honeycomb or hexagonal symmetry which will automatically introduce some hexatic order into an adsorbed liquid overlayer. Consequently, although the positional and the orientational correlations seem to follow the predicted KT evolution at the overlayer freezing transition, the role of the substrate remains an important unresolved issue.
In this paper we show that the melting transition of tilted hexatic smectic-I (Sm-I) monolayers on a liquid smectic- $C(\mathrm{Sm}-C)$ substrate is first order in five-layer FSLC films of $N$-[4-( $n$-heptyl)benzylidene]-4- $(n$ heptyl)aniline ${ }^{14}$ (7O.7) a material extensively studied in bulk $^{15}$ and in thin and thick films. ${ }^{16-21}$ Although this does not contradict the KTHNY predictions-since the theory only predicts the possibility of a two-step KT transition which can be preempted by a first-order transition-it is surprising since many of the two- and three-dimensional liquid-crystal liquid-to-hexatic transitions studied previously by other groups are continuous. $^{7-10,22}$ The melting of hexatic monolayers on a liquid substrate is an ideal way to study effectively substratefree 2D melting, since a liquid substrate will not induce any periodic positional or orientational correlations into the hexatic overlayer. However, because the $\mathrm{Sm}-C$ phase is a tilted liquid with anisotropic molecules, the coupling between the tilt and the bond orientation will, in principle, produce some hexatic order in the $S \mathrm{~m}-C$ phase ${ }^{23} \mathrm{x}$ ray scattering measurements on thick Sm- $C$ films of 70.7 have shown that the induced hexatic order is unobservably small. ${ }^{24}$ This is different from the behavior observed in thick films of 8OSI and other materials, where welldeveloped orientational order exists deep into the Sm-C phase. $^{9,10}$

The formation of Sm-I monolayers on the two filmvapor interfaces of the Sm-C interior in FSLC films of 
70.7 (Ref. 25) is an example of surface-induced order or "surface freezing." 26 Liquid crystals are prime examples of systems with surface-induced order. Many liquidcrystal systems with more ordered surface phases have been discovered, but no examples of less ordered surface phases (surface melting) have been reported. Both layerby-layer $(L)$ and continuous $(C)$ growth of the ordered surface phase into the interior have been reported; examples include: nematic order at isotropic-solid interfaces $(C),{ }^{27}$ smectic- $A$ order at the isotropic-vapor interface $(L),{ }^{28}$ smectic- $A$ order at the nematic-vapor interface (C), ${ }^{29,30} \mathrm{Sm}-C$ order at the $\mathrm{Sm}-A$-vapor interface $(C),{ }^{31}$ and crystalline- $B$ order at the $\mathrm{Sm}-A$-vapor interface $(L) .^{32}$

Surface freezing transitions are special cases of interfacial wetting ${ }^{33}$ and have been classified into three generic categories: nonwetting, incomplete wetting, and complete wetting. The freezing of most FSLC films falls into the nonwetting category: the entire film freezes abruptly. In this paper, we show that the Sm- $C$ to $\mathrm{Sm}-I$ transition in five layer 70.7 FSLC films exhibit wetting by a single surface layer: first the two exterior surface monolayers freeze and then, at a lower temperature, the rest of the interior freezes abruptly; both transitions are first order. ${ }^{34}$ The analogous surface freezing of a nontilted system (monolayer crystalline smectic- $B$ order on smectic- $A$ FSLC 14S5 films) has been reported previously by Pindak, Moncton, Bishop, and co-workers. ${ }^{32}$ Recently, "complete" layer-by-layer surface freezing (tilted Sm-I order on a nontilted smectic- $A$ interior) has been observed in 90.4 , a homolog of $70.7 .^{35}$

The hexatic-to-liquid transitions in FSLC films and in bulk samples have been studied by several groups using heat capacity, ${ }^{22}$ x-ray scattering, ${ }^{7,8}$ optical, ${ }^{9}$ electronscattering, ${ }^{10}$ and mechanical ${ }^{36}$ techniques. Both the nontilted hexatic- $B$ to smectic- $A$ and the tilted Sm- $I$ to Sm- $C$ transitions have been studied; both have continuous or weakly first-order transitions with considerable pretransitional evolution between the hexatic and liquid phases. The reported continuous hexatic- $B$ to smectic- $A$ transitions have specific-heat exponents near $\frac{1}{2}(\alpha \sim 0.48-0.67)$ (Refs. 22 and 37) and continuous evolution of the in-plane positional correlation lengths, ${ }^{7,8}$ but the critical behavior is not fully understood. ${ }^{37}$ As predicted by the KTHNY theory, the torsional oscillator measurements ${ }^{36}$ show that the hexatic- $B$ phase does not support an in-plane shear. The Sm-C to Sm-I transition is different than the smectic- $A$ to hexatic- $B$ transition because of the tilt of the molecules with respect to the smectic layers in the $\mathrm{Sm}-\mathrm{C}$ and $\mathrm{Sm}-\mathrm{I}$ phases. The tilt has several important consequences: (1) It induces some hexatic order in the Sm- $C$ phase, changing it from a tilted liquid to an induced hexatic; ${ }^{23}$ the induced order can have Sm-I, smectic- $F$ or possibly smectic- $L$ symmetry. ${ }^{38}$ (2) If the tilt-induced order has Sm- $I$ symmetry, the $\mathrm{Sm}-C$ phase is simply a Sm-I phase with smaller amplitude Sm-I order parameters and the only true phase transition between the $C$ and the $I$ must be first order. Observations of the $\mathrm{Sm}-C$ to $\mathrm{Sm}-I$ transition in bulk show a discontinuous jump in the layer spacing, which implies the transition is first order. ${ }^{39}$ The surface and interior Sm-C to Sm- $I$ tran- sitions in 70.7 reported here are true first-order transitions. (3) In the absence of a first-order transition, the tilt-induced hexatic order grows continuously, erasing the Sm- $C$ to Sm-I phase boundary. Brock et al. ${ }^{8}$ measured the growth of the amplitudes of the hexatic orientational order parameter in a system (8OSI) with continuous evolution and showed that there is a universal scaling relationship between the amplitudes. (4) The large optical contrast produced by the tilted anisotropic molecules allows the tilt domains and the characteristic defects to be imaged optically; the characteristic defects have been used by Dierker, Pindak, and Meyer ${ }^{9}$ to show the bondorientational elasticity follows the continuous KTHNY predictions at the chiral smectic- $C^{*}$ to smectic- $I^{*}$ transition. (5) The tilt distorts the hexagonal in-plane lattice into a rectangular structure ${ }^{19,40}$ so that tilted crystals are predicted to exhibit anisotropic $2 \mathrm{D}$ melting. ${ }^{41}$ In this case, there are two predicted types of critical behavior: Type II melting with the same KTHNY critical behavior
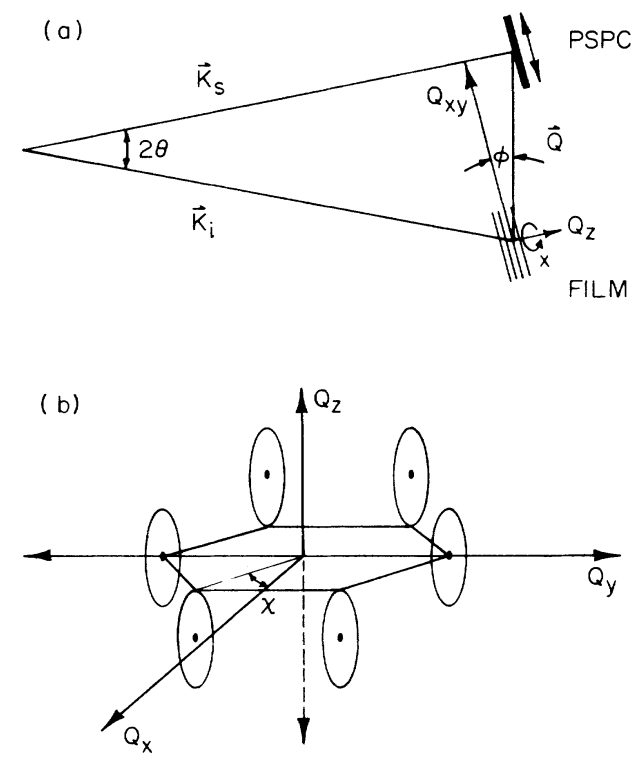

FIG. 1. (a) Schematic illustration of the scattering geometry. The position-sensitive proportional counter (PSPC) was oriented with its active direction perpendicular to the $2 \theta$ arms of the spectrometer and in the scattering plane. The scattering angle $(2 \theta)$, the incident $\left(\mathbf{K}_{i}\right)$ and scattered $\left(\mathbf{K}_{s}\right)$ wave vectors, the longitudinal $\left(\mathbf{Q}_{x y}\right)$ and transverse $\left(\mathbf{Q}_{z}\right)$ components of the momentum transfer, and the sample orientation angles $(\phi$ and $\chi)$ are shown. The spectrometer and film configuration shown produces a PSPC scan that is approximately along $\mathbf{Q}_{x y}$ with roughly constant $\mathbf{Q}_{z}$. (b) The smectic- $I$ scattering pattern for a monodomain sample. The slightly distorted solid hexagon is in the $\mathbf{Q}_{x y}$ plane. The six diffuse hexatic scattering rods are shown. Their $\left(Q_{x y}, Q_{z} ; \chi\right)$ coordinates are $\left(0.956,-2 ; \pm 30^{\circ}\right),(0.995$, $\left.0 ; \pm 90^{\circ}\right)$ and $\left(0.956,+2 ;+-120^{\circ}\right)$. The units of $Q_{x y}$ and $Q_{z}$ are described in the text. The plane containing the centers of the hexatic spots is tilted by $19.5^{\circ}$ (the director tilt) with respect to the smectic layers (the $\mathbf{Q}_{x y}$ plane). The observed scattering was the powder average (around $\mathbf{Q}_{z}$ ) of the monodomain pattern shown here. 
in both in-plane directions or type I melting with different critical behavior in the two directions. Thus, different functional dependences may hold for the two anisotropic correlation lengths over a temperature range in the Sm-I phase. Measuring the X-ray scattering from inequivalent directions in-plane at the surface $\mathrm{Sm}-I$ or interior Sm-C to Sm-C transition can establish which of these conditions holds here.

\section{EXPERIMENTAL METHOD AND RESULTS}

The $x$-ray scattering measurements were made at both the Oak Ridge National Laboratory beamline, X-14, at the National Synchrotron Light Source ${ }^{42}$ (NSLS) and at beamline VII-2 at the Stanford Synchrotron Radiation Laboratory ${ }^{43,25}$ (SSRL). At NSLS, the monochromator was a sagittally focusing $\mathrm{Si}[111]$ crystal tuned to $\lambda=1.4875 \AA$. At SSRL, a pair of asymmetrically cut $\mathrm{Ge}[111]$ crystals were used in the double-crystal monochromator to provide a well-collimated beam with $\lambda=1.7431 \AA$. Virtually identical signal counting rates were obtained at NSLS and SSRL. The scattering from the five-layer $\mathrm{Sm}-C$ films was very weak, $\sim 0.7$ counts/mm sec at the peak; this was comparable to the no-film background scattering from the apparatus. Because of the extremely low signal count rates, it was essential to use a position-sensitive proportional counter (PSPC), a Braun model OED-50, to provide parallel detection of the scattering $x$ rays. ${ }^{25}$ This provided about a 20-fold increase in the data-collection rate over a crystal analyzer ( $\mathrm{LiF}[002])$ with comparable resolution. The scattering geometry is shown in Fig. 1(a). The momentum-transfer component perpendicular to the smectic layers is $Q_{z}$ and the component in the plane parallel to the layers is $\mathbf{Q}_{x y}$. The orientation of the films around the $\mathbf{Q}_{z}$ axis was selected by the angle $\chi$. The inplane resolution was controlled by the beam width along the linear detector, which was set at the monochromator output slits. At NSLS, the monochromator output slits were set to the size of the focussed beam, $1 \times 1 \mathrm{~mm}^{2}$. At SSRL, the out-of-plane (beam height) slits were opened to $4 \mathrm{~mm}$ to accept the larger focal spot; the setting was $1 \times 4$ $\mathrm{mm}^{2}$. The $1-\mathrm{mm}$ beam width at NSLS and SSRL produced a rectangular resolution function with $\left|\Delta \mathbf{Q}_{x y}\right|=4.7 \times 10^{-3} \AA^{-1}$ full width. This resolution width corresponds to the value calculated for a $1-\mathrm{mm}$ wide beam with the PSPC located at a radius of $70 \mathrm{~cm}$ and was also directly measured by observing the Bragg scattering from a film in the crystalline- $B$ phase.

The scattering pattern for a single tilt domain hexatic Sm-I film is shown in Fig. 1(b). The nonzero molecular tilt distorts the local in-plane hexagonal packing $25,40,19$ producing two diffuse spots at $Q_{x y}=0.995, Q_{z}=0$ and four diffuse spots at $Q_{x y}=0.956, Q_{z} \simeq \pm 2$; here $Q_{x y}$ is expressed in units of $4 \pi / \sqrt{3} a$ and $Q_{z}$ in units of $2 \pi / c$, where $a=5.05 \AA$ is the hexagonal lattice parameter and $c=30.6 \AA$ is the layer spacing in the crystalline- $B$ phase. $^{16}$ Because the films consist of many small $(\sim 100 \mu \mathrm{m})$ randomly oriented tilt domains, the observed scattering pattern is the powder average of the monodomain pattern shown in Fig. 1(b). This produces two well-separated concentric cylinders of scattering. ${ }^{25}$ In each phase, the line shapes of the $Q_{z}=0$ and the $Q_{z} \simeq 2$ peaks were measured. Because of the $Q_{z} \simeq 2$ peaks were almost as wide as the $\mathrm{Sm}-C$ scattering, it was difficult to accurately determine the correlation lengths associated with these peaks. The measured $\mathrm{Sm}-C$ scattering was a powder-averaged diffuse cylinder with $Q_{x y}=0.99$ in agreement with the many small domains observed optically.
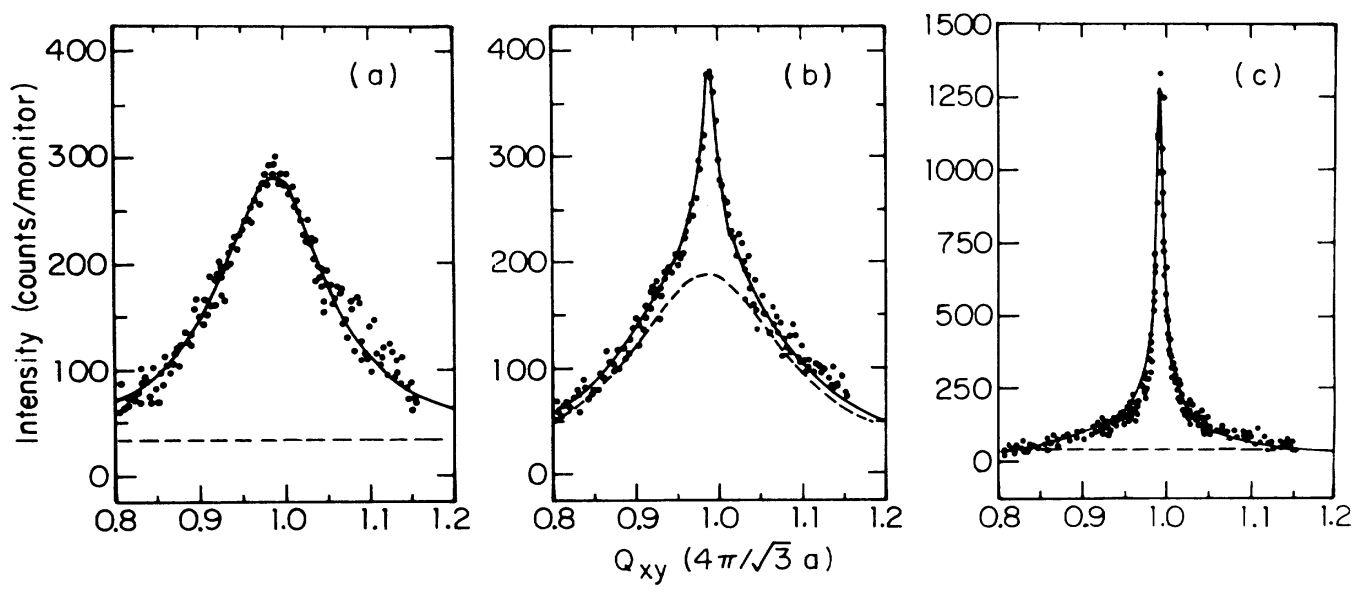

FIG. 2. Comparison of the measured scattering with the corresponding fits for the $Q_{z}=0$ spots for a five-layer film of 70.7. The data have been corrected for the no-film background and normalized to a constant incident flux. The three overlapping PSPC scans are visible. The fits are indicated by a solid line. (a) Scans taken at $78.14^{\circ} \mathrm{C}$ where the entire film is smectic $C$. As shown, the data is well fit by a single Lorentizan with $\xi=10 \AA$ plus constant diffuse background (dashed line). This scan took 35 min for all PSPC settings. (b) Scans taken at $77.67^{\circ} \mathrm{C}$ where the two surface monolayers are smectic $I$ and the three interior layers are smectic $C$. The fit shown is the sum of a broad smectic- $C$ Lorentzian (dashed line) and a narrow smectic- $I$ square-root Lorentzian (SRL). The SRL correlation length is $\xi=90 \AA$. This scan took $2 \mathrm{~h}$. (c) Scans taken at $69.09^{\circ} \mathrm{C}$ where the entire film is smectic $I$. The fit shown is the sum of a single sharp SRL with $\xi=260 \AA$ plus a small constant diffuse level (dashed line). This scan took $2 \mathrm{~h}$. 
The film oven used for these measurements has been described in detail previously. ${ }^{18,20}$ Cobalt samarium magnets were installed inside the oven on two opposing sides of the film to provide $\mathrm{a} \sim 1 \mathrm{kG}$ field to orient the tilt domains. Although this configuration produced wellaligned bulk $\mathrm{Sm}-\mathrm{C}$ films of other liquid crystals, ${ }^{24}$ it did not produce well-aligned $\mathrm{Sm}-C$ or $\mathrm{Sm}-I$ surface monolayers in 70.7. As soon as the films were cooled from the smectic- $A$ phase into the surface phase, many small tilt domains were produced; these domains were observed optically and also produced x-ray $\chi$ scans (at fixed $Q_{x y}$ and $Q_{z}$ ) which were independent of $\chi$.

All of the scans reported here are for five-layer films of 70.7 with the no-film background subtracted from the raw data and the measured scattering corrected for incident beam intensity changes. Figure 2 shows typical scans for the $Q_{x y}=0.995, Q_{z}=0$ scattering in the Sm-C, surface Sm-I and interior Sm-C, and Sm-I phases. Because the PSPC is not wide enough to cover the broad $\mathrm{Sm}-C$ peaks, each scan shown is a composite of data collected at three PSPC positions with overlapping $\mathbf{Q}_{x y}$ values and essentially identical $Q_{z}$ values. As shown in Fig. 2(a), the measured Sm-C scattering was fit very well by a single Lorentzian peak added to a constant diffuse background. The fitted Lorentzian widths were essentially independent of temperature in the $\mathrm{Sm}-C$ phase. The scattering from the surface Sm-I films [see Fig. 2(b)] was fit very well by the sum of a square-root Lorentzian (SRL),

$$
S\left(\mathbf{Q}_{x y}\right)=A \xi\left[1+\left(\mathbf{Q}_{x y}-\mathbf{Q}_{0}\right)^{2} \xi^{2}\right]^{-1 / 2},
$$

which represents the surface $\mathrm{Sm}-I$ scattering contribution and a Lorentzian which represents the interior Sm- $C$ contribution. The SRL form arises because the monodomain Sm-I peaks are assumed to be Lorentzians; powder averaging them in the azimuthal direction results in a form which is approximately a SRL. ${ }^{44}$ The fitted Lorentzian widths for the interior $\mathrm{Sm}-C$ phase were independent of the temperature and were identical in width to the five-layer Sm-C fits. The total scattering from the surface Sm-I/interior Sm-C phase in Fig. 2(b) could not be fit by either a single Lorentzian or by a single SRL. The scattering when the entire film is $\mathrm{Sm} I$ is as shown in Fig. 2(c). As shown this data was fit very well by a single SRL plus a constant diffuse background.

The $Q_{x y}=0.956, Q_{z} \simeq \pm 2$ data was analyzed in the same way: Lorentzian Sm-C scattering, SRL surface- $I$ scattering, and SRL Sm-I interior scattering. However, because the $Q_{z} \simeq \pm 2$ surface Sm- $I$ peaks were about as wide as the $\mathrm{Sm}-C$ peaks, it was more difficult to determine the surface and interior widths reliably.

The SRL parameters versus temperature obtained from the nonlinear least-square fits to the functions described above are shown in Fig. 3. These fits determined the SRL scattering amplitude $A_{s}$, which is proportional to the integrated intensity of the Sm-I scattering, and the SRL correlation length $\xi_{s}$, which is proportional to the inverse of the width of the Sm-I peak. Complete data sets taken on cooling and heating are shown; other scans (not shown) taken close to the transition at $\sim 77.7$ and

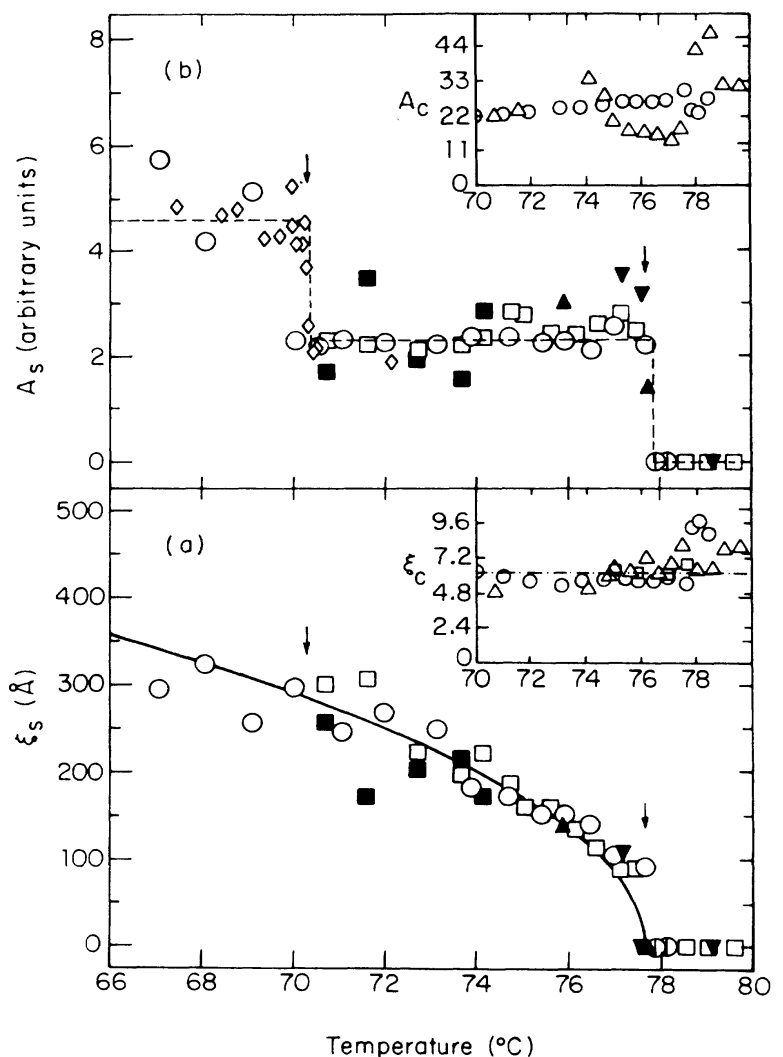

FIG. 3. Square-root-Lorentzian (SRL) scattering amplitude $A_{s}$ and correlation length $\xi_{s}$ determined by the nonlinear leastsquare fits for the $Q_{z}=0$ and $Q_{z} \simeq 2$ peaks. The open and closed symbols represent $Q_{z}=0$ and $Q_{z} \simeq 2$ data, respectively. The open circles and diamonds represent data taken upon cooling at NSLS; the open squares and triangles (upright and inverted) represent, respectively, the cooling and heating data taken at SSRL. The $Q_{z} \simeq 2$ data are plotted as the corresponding filled symbols. The monolayer surface transition at $\sim 77.7^{\circ} \mathrm{C}$ and the interior transition at $\sim 70.3^{\circ} \mathrm{C}$ are indicated by the arrows. (a) The SRL scattering amplitudes, $A_{s}$ vs temperature. This amplitude is proportional to the number of smectic- $I$ layers. The dashed line represents the average value of $\boldsymbol{A}_{s}$ in each phase: $A_{s} \sim 0$ in the smectic $C, A_{s} \sim 2.3$ in the surface Sm- $I$ and interior Sm-C, and $A \sim 4.6$ in the smectic $I$. The $Q_{z} \simeq 2$ SRL amplitudes are also shown; they have been multiplied by $\frac{2}{3}$ to coincide with the $Q_{z}=0$ data in the surface Sm- $I$ and interior Sm-C phase. (b) The SRL correlation length $\xi_{s}$ vs temperature. In the smectic- $C$ phase the SRL amplitude, $A_{s} \sim 0$, and the SRL correlation length $\xi_{s}$ are also zero accordingly. There is a firstorder jump in $\xi_{s}$ at the surface $\operatorname{Sm} I$ transition followed by a roughly square-root evolution, $\xi_{s} \sim\left|T-T_{C}\right|^{1 / 2}$ (solid line). The $Q_{z} \simeq 2$ data has been multiplied by 2.6 to correspond with the jump in the $Q_{z}=0$ data. When scaled in this way, the data for both $Q_{z}$ values exhibit the same temperature evolution within the measured errors. No variation in $\xi_{s}$ is visible at the surface $\mathrm{Sm}-I$ and interior Sm- $C$ to smectic- $I$ transition. Insets: The smectic- $C$ Lorentzian amplitudes $A_{C}$, (a) and correlation lengths $\xi_{C}$, (b) vs temperature. For the fits to the surface $\mathrm{Sm}-I$ and interior Sm- $C$ data,$\xi_{C}$ was fixed at the value indicated by the dashed line. 
$\sim 70.5^{\circ} \mathrm{C}$ indicated that both were hysteretic. Above about $78^{\circ} \mathrm{C}$, the films were $\operatorname{Sm} C$ and $A_{s} \sim 0$. The insets to Fig. 3 show the evolution of the Sm- $C$ Lorentzian amplitudes $A_{c}$ and correlation lengths $\xi_{C}$. The latter are approximately constant throughout the surface $\mathrm{Sm}-\mathrm{I}$ and interior $\mathrm{Sm}-\mathrm{C}$ phase. Just below $78^{\circ} \mathrm{C}$, the two monolayers at each film-vapor interface freeze, forming surface Sm- $I$ monolayers on the Sm- $C$ interior producing a discontinuous jump in the SRL amplitudes and the SRL correlation length. Within the surface Sm-I and interior Sm- $C$ phase $\xi_{s}$ increases from $\sim 100$ to $\sim 275 \AA$ while $A_{s}$ and the Sm- $C$ peak height remain roughly constant as the temperature is reduced from 78 to $70.5^{\circ} \mathrm{C}$. At about $70.5^{\circ} \mathrm{C}$ the interior layers freeze and the entire film is $\mathrm{Sm}$ $I$. There is a corresponding jump in the $\mathrm{Sm}-\mathrm{I}$ amplitude, but the correlation length does not have any observable jump. The ratio of the SRL amplitude in the Sm-I phase to the surface SRL amplitude in the surface Sm- $I$ and interior $\mathrm{Sm}-C$ phase is about $4.6: 2.3$. This is in reasonable agreement (within the experimental errors) with the ratio of the film thicknesses (five layers) to the surface thickness (two layers). The observed discontinuous jumps at the surface and interior freezing transitions show they are both first-order phase transitions. In addition, both transitions were also slightly hysteretic $\left(\sim 0.2^{\circ} \mathrm{C}\right)$.

The evolution in the surface correlation length after the first-order transition to the surface $\mathrm{Sm}-I$ phase at $\sim 78^{\circ} \mathrm{C}$ is consistent with a square-root scaling law, $\xi_{s} \sim\left|T-T_{C}\right|^{1 / 2}$, but the uncertainty in $\xi_{s}$ is quite large both because of the low counting rates and also because of the difficulty in separating the interior Sm-C and the surface Sm-I scattering. Figure 3 also compares the amplitude and surface correlation length evolution for the $Q_{z}=0$ and $Q_{z} \simeq 2$ peaks with each other and with the square-root form. Since the $Q_{z} \simeq 2$ peak is wider than the $Q_{z}=0$ peak, the $Q_{z} \simeq 2$ correlation lengths were scaled by a multiplicative constant $\left(\frac{1}{2.6}\right)$ to make the first-order jumps at the surface freezing transition equal. When scaled in this way, the evolution with temperature is the same within the errors. Consequently, there is no evidence for anisotropic type I evolution above the firstorder jump at $70.5^{\circ} \mathrm{C}$ in this system. This isotropic evolution is also different from the reported evolution in 8OSI (Ref. 8) which is anisotropic.

\section{CONCLUSIONS}

In summary, we have shown that the monolayer surface hexatic Sm-I layers in freely suspended films of 70.7 melt via a hysteretic first-order transition into the $\mathrm{Sm}-\mathrm{C}$ phase. Since the Sm- $C$ phase is predicted to have induced hexatic (presumably $\mathrm{Sm}-I$ ) order, this transition is a novel first-order hexatic-to-hexatic monolayer surface freezing transition in which the symmetry of the phase is un- changed, but the amplitude of the order parameters jump discontinuously. The microscopic mechanism for this first-order jump without any change in the symmetry is an unresolved problem. The observed first-order jump is consistent with the recent suggestion ${ }^{45}$ that there is a nearby tricritical point in all Sm- $C$ to Sm- $I$ to Sm- $J$ transitions. In addition, we see no evidence for any pretransitional evolution on the $\mathrm{Sm}-C$ side of the transition where the previous studies in systems with continuous evolution show large growth of the induced order. This suggests that the coupling between the tilt and the bondorientational and positional order is much weaker in 70.7 than in 8 OSI or that there is an unusual microscopic mechanism which suddenly produces a large induced hexatic response over a small temperature interval.

We also find that the growth and disappearance of the hexatic Sm-I order in the interior of the film-the Sm-C interior freezing and melting transitions - are hysteretic first-order transitions. In surface freezing and wetting language this puts the transition in the incomplete wetting category: the first hexatic monolayer wets the Sm$C$-vapor interface, but the successive hexatic layers do not. The origin of the very different surface freezing behavior in different FSLC films is also an interesting open problem: Why in some systems $(70.7, \overline{14 S 5})$ does only the first monolayer wet while in others (9O.4) hundreds of layers wet? Finally, it is an open question why there is no surface-induced order in the materials (e.g., 8OSI) which evolve continuously.

\section{ACKNOWLEDGMENTS}

We would like to thank the support staff at SSRL and NSLS for their help. This work was supported by the National Science Foundation through Grant Nos. DMR-85-13523 (to P.S.P.) and DMR-86-12286 (to L.B.S.) and a grant to the Harvard Materials Research Laboratory, NSF-DMR-86-14003. The synchrotron measurements were carried out in part at the Oak Ridge National Laboratory Beamline X-14 at the National Synchrotron Light Source at Brookhaven National Laboratory sponsored by the Division of Material Sciences and the Division of Chemical Sciences, U.S. Department of Energy, under Contract No. DE-AC05-840R21400 with Martin Marietta Energy Systems, Inc. The National Synchrotron Light Source is supported by the Department of Energy, Material Sciences and Division of Chemical Sciences under Contract No. DE-AC02-76CH00016. Other synchrotron measurements were made at the Stanford Synchrotron Radiation Laboratory at the Stanford Linear Accelerator Laboratory, which is supported by the Department of Energy Office of Basic Energy Sciences and the National Institutes of Health Biotechnology Research Program, Division of Research Resources.
${ }^{1}$ B. I. Halperin and D. R. Nelson, Phys. Rev. Lett. 41, 121 (1978); 41, 519(E) (1978); D. R. Nelson and B. I. Halperin, Phys. Rev. B 19, 2457 (1974); A. P. Young, ibid. 19, 1855 (1979).
${ }^{2}$ For a very recent review see K. J. Strandburg, Rev. Mod. Phys. 60, 161 (1988).

${ }^{3}$ For earlier reviews see D. R. Nelson, in Phase Transitions and Critical Phenomena, Vol. 7, edited by C. Domb and J. L. Le- 
bowitz (Academic, New York, 1983); S. Ostlund, J. Toner, and A. Zippelius, Ann. Phys. (N.Y.) 144, 345 (1982).

${ }^{4}$ J. M. Kosterlitz and D. J. Thouless, J. Phys. C 6, 1181 (1973); in Progress in Low Temperature Physics, edited by D. F. Brewer (North-Holland, Amsterdam, 1978), Vol. VII-B.

${ }^{5}$ For a recent review see R. J. Birgeneau and P. M. Horn, Science 232, 329 (1986); also see N. Greiser, G. A. Held, R. Frahm, R. L. Green, P. M. Horn, and R. M. Suter, Phys. Rev. Lett. 59, 1705 (1987), and references therein.

${ }^{6}$ C. A. Murray and D. H. Van Winkle, Phys. Rev. Lett. 58, 1200 (1987).

${ }^{7}$ S. C. Davey, J. Budai, J. W. Goodby, R. Pindak, and D. E. Moncton, Phys. Rev. Lett. 53, 2129 (1984).

${ }^{8}$ J. D. Brock, A. Aharony, R. J. Birgeneau, K. W. EvansLutterodt, J. D. Litster, P. M. Horn, G. B. Stephenson, and A. R. Tajbarkhsh, Phys. Rev. Lett. 57, 98 (1986).

${ }^{9}$ S. B. Dierker, R. Pindak, and R. B. Meyer, Phys. Rev. Lett. 56, 1819 (1986).

${ }^{10}$ M. Cheng, J. T. Ho, S. W. Hui, and R. Pindak, Phys. Rev. Lett. 59, 1112 (1987).

${ }^{11}$ S. T. Chui, Phys. Rev. Lett. 48, 933 (1982); Phys. Rev. B 28, 178 (1983).

${ }^{12}$ Y. Saito, Phys. Rev. Lett. 48, 1114 (1982); Phys. Rev. B 26, 6239 (1982).

${ }^{13}$ H. K. Kleinert, Phys. Lett. A 95, 381 (1983).

${ }^{14}$ Our 7 O.7 samples were supplied by CPAC Organix, Inc.

${ }^{15}$ J. Doucet and A. M. Levelut, J. Phys. (Paris) 38, 1163 (1977).

16J. Collett, L. B. Sorenson, P. S. Pershan, J. D. Litster, R. J. Birgeneau, and J. Als-Nielsen, Phys. Rev. Lett. 49, 553 (1982).

${ }^{17} \mathrm{~J}$. Collett, P. S. Pershan, E. B. Sirota, and L. B. Sorensen, Phys. Rev. Lett. 52, 356 (1984).

${ }^{18}$ J. Collett, L. B. Sorensen, P. S. Pershan, and J. Als-Nielsen, Phys. Rev. A 32, 1036 (1985).

${ }^{19}$ E. B. Sirota, P. S. Pershan, L. B. Sorensen, and J. Collett, Phys. Rev. Lett. 55, 2039 (1985).

${ }^{20}$ E. B. Sirota, P. S. Pershan, L. B. Sorensen, and J. Collett, Phys. Rev. A 36, 2890 (1987).

${ }^{21}$ E. B. Sirota, P. S. Pershan, and M. Deutsch, Phys. Rev. A 36, 2902 (1987).

${ }^{22}$ C. C. Huang, G. N. Nounesis, and D. Guillon, Phys. Rev. A 33, 2602 (1986); T. Pitchford, G. Nounesis, S. Dumrongrattana, J. M. Viner, C. C. Huang, and J. W. Goodby, Phys. Rev. A 28, 2433 (1983); C. C. Huang, J. M. Viner, R. Pindak, and J. W. Goodby, Phys. Rev. Lett. 46, 1289 (1981).

${ }^{23}$ D. R. Nelson and B. I. Halperin, Phys. Rev. B 21, 5312 (1980).

${ }^{24}$ J. A. Collett, Ph.D. thesis, Harvard University, 1983.

${ }^{25}$ E. B. Sirota, P. S. Pershan, S. Amador, and L. B. Sorensen, Phys. Rev. A 35, 2283 (1987).

${ }^{26}$ R. Lipowsky, J. Appl. Phys. 55, 2485 (1986); Ferroelectrics 73, 69 (1987); also see the recent review on surface freezing by $\mathbf{J}$. G. Dash, in Proceedings XIX Solvay Conference, edited by F. W. Dewitte (Springer-Verlag, New York, 1988).

${ }^{27}$ For a comprehensive review of both experimental and theoretical work on surface-induced order in nematic liquid crystals, see T. J. Sluckin and A. Poniewierski, in Fluid Interfacial Phenomena, edited by C. A. Croxton (Wiley, New York,
1986); for recent experimental work see H. Hsiung, Th. Rasing, and Y. R. Shen, Phys. Rev. Lett. 57, 3065 (1986); 59, 1983(E) (1987).

${ }^{28}$ B. M. Ocko, A. Braslau, P. S. Pershan, J. Als-Nielsen, and M. Deutsch, Phys. Rev. Lett. 57, 94 (1986); J. Als-Nielsen, Physica 140A, 376 (1986); Z. Pawlowska, G. F. Kventsel, and T. J. Sluckin, Phys. Rev. A 36, 992 (1987); J. V. Selinger and D. R. Nelson, ibid. 37, 1736 (1988).

${ }^{29}$ J. Als-Nielsen, F. Christensen, and P. S. Pershan, Phys. Rev. Lett. 48, 1107 (1982); J. Als-Nielsen and P. S. Pershan, Nucl. Instrum. Methods 208, 545 (1983); P. S. Pershan and J. AlsNielsen, Phys. Rev. Lett. 52, 759 (1984).

${ }^{30}$ Also see the review by J. Als-Nielsen, in Structure and Dynamics of Surfaces II, edited by $\mathrm{W}$. Schommers and $\mathrm{P}$. Blanckenhagen (Springer-Verlag, New York, 1987).

${ }^{31}$ S. Heinekamp, R. A. Pelcovits, E. Fontes, E. Y. Chen, R. Pindak, and R. B. Meyer, Phys. Rev. Lett. 52, 1017 (1984).

${ }^{32}$ D. J. Bishop, W. O. Sprenger, R. Pindak, and M. E. Neubert, Phys. Rev. Lett. 49, 1861 (1982); D. E. Moncton, R. Pindak, S. C. Davey, and G. S. Brown, ibid. 49, 1865 (1982).

${ }^{33}$ G. An and M. Schick, Phys. Rev. B 37, 7534 (1988).

${ }^{34}$ Independent optical studies show that this same monolayer surface freezing occurs for films up to hundreds of layers in thickness. L. B. Sorensen, H. Stragier, B. D. Swanson, and D. J. Tweet (unpublished).

${ }^{35}$ B. D. Swanson, H. Stragier, D. J. Tweet, and L. B. Sorenson (to be published). The $\mathrm{Sm}-\mathrm{I}$ phase in 90.4 is a surface stabilized phase (it does not exist in bulk) and therefore the wetting will not be complete in a semi-infinite system. However, the freezing proceeds in a layer-by-layer fashion until the entire film is frozen in finite-thickness FSLC films. The limiting thickness is not known, but is over 100 layers.

${ }^{36}$ R. Pindak, W. O. Sprenger, D. J. Bishop, D. D. Osheroff, and J. W. Goodby, Phys. Rev. Lett. 48, 173 (1982).

${ }^{37}$ G. Nounesis, R. Greer, H. Y. Liu, C. C. Huang, and J. W. Goodby (unpublished).

${ }^{38}$ G. S. Smith, E. B. Sirota, C. R. Safinya, and N. A. Clark, Phys. Rev. Lett. 60, 813 (1988); J. V. Selinger and D. R. Nelson (unpublished).

${ }^{39}$ See, for example, S. Kumar, Phys. Rev. A 23, 3207 (1981).

${ }^{40}$ J. Doucet, P. Keller, A. M. Levelut, and P. Porquet, J. Phys. (Paris) 39, 548 (1978); P. A. C. Gane, A. J. Leadbetter, and P. G. Wrighton, Mol. Cryst. Liq. Cryst. 66, 245 (1981); J. J. Benattar, F. Moussa, M. Lambert, and C. Germain, J. Phys. (Paris) Lett. 42, 67 (1981); J. J. Benattar, F. Moussa, and M. Lambert, J. Chem. Phys. 80, 99 (1983).

${ }^{41}$ S. Ostlund and B. Halperin, Phys. Rev. B 23, 335 (1981).

${ }^{42}$ A. Habenschuss, G. E. Ice, C. J. Sparks, and R. A. Neiser, Nucl. Instrum. Methods A266, 215 (1988).

${ }^{43}$ D. E. Moncton and G. S. Brown, Nucl. Instrum. Methods 208, 576 (1983).

${ }^{44}$ B. M. Ocko, A. R. Kortan, R. J. Birgeneau, and J. W. Goodby, J. Phys. (Paris) 45, 113 (1984).

${ }^{45}$ A. A harony, R. J. Birgeneau, J. D. Brock, and J. D. Litster, Phys. Rev. Lett. 57, 1012 (1986). 